\title{
An unsere Mitarbeiter und Leser
}

Einem Wunsche der Pathologischen Anatomen unseres Her-ausgeber-Kollegiums entsprechend, haben wir den durch dieKriegs-verhältnisse bedingten Störungen im Publikationswesen dadurch Rechnung getragen, daß wir 1941 die Spalten der Schweiz. Zeit-schrift für Allgemeine Pathologie und Bakteriologie auch der speziellen pathologischen Anatomie zugänglich machten. Das be-dingte naturgemäß einen erheblichen Zustoß an Arbeiten und da-mit eine unliebsame Verlängerung der Erscheinungszeiten. Wir suchten 1947 das Problem durch eine Vermehrung des Umfanges von 4 auf 6 Bogen zu meistern, erreichten aber auch dadurch die gewünschte Entlastung nicht.

Mit Rücksicht auf die Tatsache, daß die führenden deutschen Zeitschriften für spezielle Pathologie wieder erscheinen, haben wir uns deshalb entschlossen, zur ursprünglichen Konzeption einer Zeitschrift für Allgemeine Pathologie und Bakteriologie zurück-zukehren, «ohne dabei» - wie wir im Vorwort zum ersten Jahr-gang betonten - «auf einer lehrbuchmäßigen Trennung von all-gemeiner und spezieller Forschung zu bestehen, die sich ja auf beiden Gebieten aus Gründen, die in der Forschung selbst gelegen sind, gar nicht durchführen läßt». Urn unsern Mitarbeitern noch weiter entgegenkommen zu können, haben wir trotz des damit verbundenen neuerlichen Preis-aufschlages mit dem Verlag vereinbart, den Umfang der Zeitschrift nochmals zu erhöhen und sie ab 1. Januar 1950 im Umfang von 8 statt 6 Bogen pro Heft erscheinen zu lassen. Wir hoffen, damit die Wartefristen auf ein erträgliches Maß reduzieren zu können, bitten aber unsere Mitarbeiter nichtsdestoweniger, im gegenseitigen Interesse die Manuskripte so kurz wie möglich zu halten.

Die Herausgeber: A. von Albertini A. Grumbach H. Mooser. Pathologie und Bakteriologie, Vol. XII, Fasc. 6 (1949) 42 